\title{
A Diagnostic Conundrum of Distributive Shock: Autoimmune Polyglandular Syndrome Type II
}

\author{
Monisha P Kumar ${ }^{1}$, Braghadheeswar Thyagarajan², Nairmeen Haller ${ }^{3}$, Daniela Ciltea ${ }^{4}$
}

\begin{abstract}
Autoimmune polyglandular syndrome (AIPS) is a heterogeneous condition characterized by the loss of immune tolerance and resultant dysfunction of multiple endocrine organs. Although this condition is insidious in nature, it frequently presents initially as adrenal insufficiency (Al). For patients in shock, physicians routinely assess for infections, volume depletion as well as cardiogenic and iatrogenic causes of shock. However, the case described in this report emphasizes the need for high suspicion of Al syndrome when the etiology of shock remains unclear after primary assessment. A subsequent evaluation for autoimmune etiology, especially in young adults in appropriate clinical setting, may also be warranted.

Keywords: Adrenal, Autoimmune, Polyglandular syndrome, Shock.

Indian Journal of Critical Care Medicine (2019): 10.5005/jp-journals-10071-23297
\end{abstract}

\section{INTRODUCTION}

Autoimmune polyglandular syndrome (AIPS) is a rare condition characterized by circulating autoantibodies and resultant lymphocytic infiltration and dysfunction of various endocrine glands. ${ }^{1}$ The objective of this report is to describe a case of AIP type II (AIPS-II) with principal manifestation of adrenal insufficiency (AI), which was successfully treated despite delayed diagnosis. Although $\mathrm{Al}$ is an uncommon condition that typically leads to distributive shock, early recognition and management play crucial roles in reducing mortality.

\section{Case Description}

A 20-year-old female, with no significant past medical history, presented to the emergency department with a myriad of complaints of abdominal pain, nausea, nonbloody, nonbilious vomiting, and fever for 5-day duration. The abdominal pain was diffuse, dull aching in nature, and associated with occasional burning. She denied any recent travel, sick contacts, or change in diet habits. Review of system was positive for poor appetite, dizziness while standing up, palpitations, myalgia, and excessive fatigue but negative for diarrhea, productive cough, dysuria, vaginal discharge, or neck stiffness. Her initial vital signs were pertinent for hypotension with a blood pressure of $73 / 33 \mathrm{~mm} \mathrm{Hg}$, and tachycardia with a heart rate of 118 per minute. She did not require supplemental oxygen and had a temperature of $37.8^{\circ} \mathrm{C}$. Physical examination revealed dry oral mucosa and mild diffuse tenderness to abdominal palpation, with no rash, ulcer, or discoloration of skin. Other physical examination findings were unremarkable.

Her lab work was significant for a white blood cell count of 9.9 (3.9-10.0) thou $/ \mathrm{mm}^{3}$, serum creatinine of $1.31(0.51-0.95) \mathrm{mg} / \mathrm{dL}$, mild hyponatremia with a sodium of $130(136-146) \mathrm{mEq} / \mathrm{L}$, potassium of $5.0(3.5-5.1) \mathrm{mEq} / \mathrm{L}$, and serum blood glucose of 63 (70-99) mg/dL. Lipase was normal and liver function test was unremarkable. She was started on aggressive fluid resuscitation, broad-spectrum intravenous (IV) antibiotics, and admitted to the medical intensive care unit for management of shock secondary to sepsis from infectious gastroenteritis and volume depletion.

Despite fluid resuscitation, the patient's mean arterial blood pressure continued to decline, hence she was started on a
1,3,4 Department of Internal Medicine, Cleveland Clinic Akron General, Akron, Ohio, USA

${ }^{2}$ Department of Critical Care Medicine, Wake Forest Baptist Medical Center, Winston-Salem, North Carolina, USA

Corresponding Author: Monisha P Kumar, Department of Internal Medicine, Cleveland Clinic Akron General, Akron, Ohio, USA, Phone: +1330-888-3913, e-mail: dr.mpdk@gmail.com

How to cite this article: Kumar MP, Thyagarajan B, Haller N, Ciltea D. A Diagnostic Conundrum of Distributive Shock: Autoimmune Polyglandular Syndrome Type II. Indian J Crit Care Med 2019;23(12): 582-583.

Source of support: Nil

Conflict of interest: None

vasopressor medication. A computed tomography of the abdomen with IV contrast was unremarkable, and no source of infection was identified on workup. However, the patient continued to require increasing vasopressor support. On hospital day 2, the patient had no episodes of vomiting and remained afebrile, with no leukocytosis. Given profound shock despite intravascular volume resuscitation and no clear identifiable source for sepsis, a morning serum cortisol level was ordered for suspected Al.

Cortisol testing was significantly low at $<0.5(10-20) \mu \mathrm{g} / \mathrm{dL}$, suggestive of Al. Further testing showed a subnormal response to low-dose adrenocorticotropic hormone (ACTH) stimulation, confirming primary Al (PAI). She was started on hydrocortisone and the patient was able to be weaned off vasoactive medication infusion along with clinical improvement in symptoms. For further evaluation of the etiology of the adrenal crisis, magnetic resonance imaging of the abdomen was ordered, which showed atrophic bilateral adrenal glands (Fig. 1).

On further evaluation, her 21-hydroxylase antibody (Ab) was significantly elevated at $78.1(0.0-1.0) \mathrm{U} / \mathrm{mL}$, suggestive of autoimmune adrenalitis. Subsequent workup for additional autoimmune conditions revealed profoundly elevated thyroglobulin $\mathrm{Ab}$ at $109.6(0.0-60.0) \mathrm{IU} / \mathrm{mL}$ and thyroid peroxidase $\mathrm{Ab}$ at 528.7 $(0.0-60.0) \mathrm{IU} / \mathrm{mL}$, with normal islet cell $\mathrm{Ab}$ and glutamic acid decarboxylase Ab level. Taken together, it was concluded that the

(c) The Author(s). 2019 Open Access This article is distributed under the terms of the Creative Commons Attribution 4.0 International License (https://creativecommons. org/licenses/by-nc/4.0/), which permits unrestricted use, distribution, and non-commercial reproduction in any medium, provided you give appropriate credit to the original author(s) and the source, provide a link to the Creative Commons license, and indicate if changes were made. The Creative Commons Public Domain Dedication waiver (http://creativecommons.org/publicdomain/zero/1.0/) applies to the data made available in this article, unless otherwise stated. 


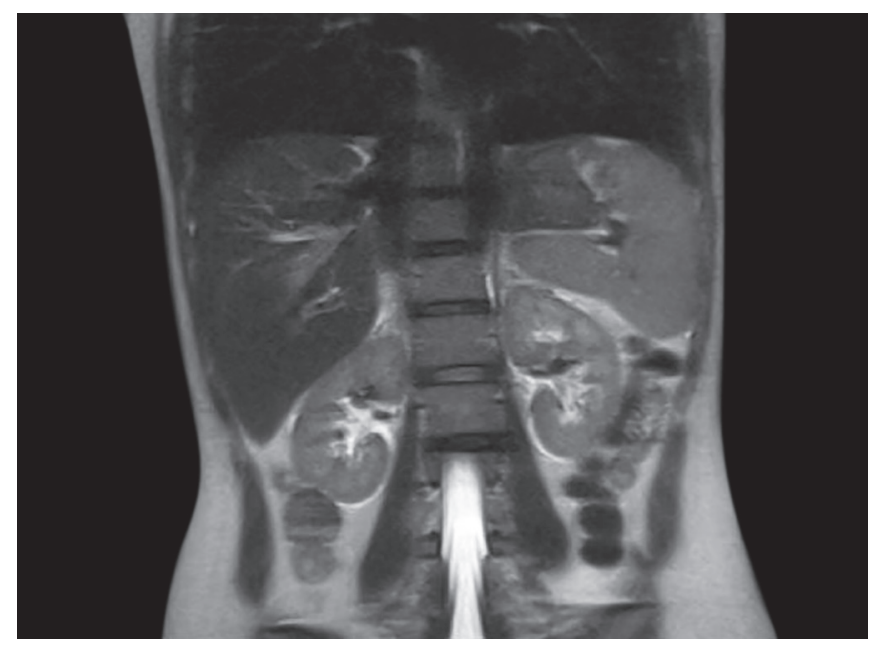

Fig. 1: Magnetic resonance image of adrenal gland demonstrating atrophic adrenal glands

patient developed shock secondary to adrenal crisis, and she was diagnosed with AIPS-II. Despite the presence of antithyroid peroxidase autoantibodies, her thyroid function tests remained normal; therefore, no thyroid replacement therapy was started. She was discharged on day 7 on a daily glucocorticoid (hydrocortisone $20 \mathrm{mg}$ with breakfast and $5 \mathrm{mg}$ with dinner) and mineralocorticoid (fludrocortisone $0.5 \mathrm{mg}$ once daily) replacement regimen, with education on sick-day steroid use rules. The patient has close follow-up with an endocrinologist and her disease is currently well controlled.

\section{Discussion}

In 1926, Martin Benno Schmidt, a German pathologist, first identified an autoimmune disease process affecting more than one endocrine organ. ${ }^{2}$ He described two cases of autoimmunity simultaneously affecting the adrenal gland and thyroid gland, and the condition of "Schmidt syndrome" was named after him. ${ }^{3}$ Upon further study, an increased understanding of the underlying disease process led to the discovery of a host of autoantibodies simultaneously affecting multiple glandular organs. This condition was named "autoimmune polyglandular syndrome" by Neufeld et al. in $1980 .{ }^{1}$

In general, AIPS is classified into three forms as AIPS-I, AIPS-II, and X-linked polyendocrinopathy. ${ }^{4}$ The AIPS-I is a rare condition with disease onset in infancy, whereas AIPS-II manifests anywhere from childhood through adulthood. ${ }^{5}$ The prevalence of AIPS-II is reported as 1:20,000 in the general population, with a 3:1 preponderance in females. ${ }^{6}$ The AIPS-II follows polygenic inheritance, and human leukocyte antigen DQ2 (HLA-DQ2) and human leukocyte antigen DQ8 (HLA-DQ8) are the commonly associated genotypes. ${ }^{5}$ As in the case described here, a majority of these patients present with adrenal crisis. ${ }^{7,8}$

The clinical presentation of adrenal crisis encompasses initial nonspecific symptoms such as nausea, vomiting, fever, anorexia, and abdominal pain, as found in this case, which mimics several other conditions including sepsis and gastrointestinal disease. The generality of the presenting symptoms poses difficulty, and often delays, in diagnosis. Oftentimes, patients with cortisol deficiency develop hyperpigmentation due to increased proopiomelanocortin and subsequent high ACTH and melanocyte-stimulating hormone, which is a clinical clue that may aid in early diagnosis. The case described here is unusual in that the patient did not have any of the characteristic skin changes. ${ }^{9}$
Concomitant aldosterone deficiency is common in patients with AIPS-II and usually leads to volume depletion and hypotension. ${ }^{10}$ Additionally, glucocorticoids are essential for epinephrine secretion by the adrenal medulla as a stress response. ${ }^{11}$ As such, Al may lead to life-threatening hypotension, making early identification and treatment with corticosteroid replacement critical in these patients. ${ }^{12}$ The patient in this report displayed persistent hypotension despite fluid resuscitation, which prompted further evaluation of other causes of shock subsequently leading to the final diagnosis of AIPS-II.

\section{Conclusion}

In summary, this report describes a case of shock in a young female secondary to adrenal crisis with underlying AIPS-II, which underscores the importance of early suspicion for $\mathrm{Al}$ in cases of resistant distributive shock in young individuals. Early identification and initiation of treatment through corticosteroid replacement will lead to decreased mortality and more rapid recovery and serve to reduce the length of stay and the overall healthcare costs. Additionally, educating patients with AIPS-II on medication compliance and sick-day rule for steroid use is of paramount importance in managing $\mathrm{Al}$ and preventing future adrenal crisis.

\section{References}

1. Sastre-Garriga J, Tintore M, Montalban X.Polyglandular autoimmune syndrome type II and multiple sclerosis. J Neurol 2001;248(4):330-331. DOI: $10.1007 /$ s004150170210.

2. Gupta AN, Nagri SK. Schmidt's syndrome - case report. Australas Med J 2012;5(6):292-295. DOI: 10.4066/AMJ.2012.987.

3. McCombe PA, Chalk JB, Pender MP. Familial occurrence of multiple sclerosis with thyroid disease and systemic lupus erythematosus. J Neurol Sci 1990;97(2-3):163-171. DOI: 10.1016/0022-510x(90)90215-9.

4. Michels AW, Gottlieb PA. Autoimmune polyglandular syndromes. Nat Rev Endocrinol 2010;6(5):270-277. DOI: 10.1038/nrendo.2010.40.

5. Eisenbarth GS. Autoimmune polyendocrine syndromes. Adv Exp Med Biol 2004;552:204-218.

6. Forster G, Krummenauer F, Kuhn I, Beyer J, Kahaly G. Polyglandular autoimmune syndrome type II: epidemiology and forms of manifestation. Dtsch Med Wochenschr 1999;124(49):1476-1481. DOI: 10.1055/s-2008-1035684.

7. Dittmar M, Kahaly GJ. Polyglandular autoimmune syndromes: immunogenetics and long-term follow-up. J Clin Endocrinol Metab 2003;88(7):2983-2992. DOI: 10.1210/jc.2002-021845.

8. Trence DL, Morley JE, Handwerger BS. Polyglandular autoimmune syndromes. Am J Med 1984;77(1):107-116. DOI: 10.1016/00029343(84)90444-3.

9. Raff H, Sharma ST, Nieman LK. Physiological basis for the etiology, diagnosis, and treatment of adrenal disorders: Cushing's syndrome, adrenal insufficiency, and congenital adrenal hyperplasia. Compr Physiol 2014;4(2):739-769. DOI: 10.1002/cphy.c130035.

10. Arai K, Chrousos GP. Aldosterone deficiency and resistance. In: De Groot LJ, Chrousos G, Dungan K, Feingold KR, Grossman A, Hershman JM, et al. ed. Endotext. South Dartmouth (MA); 2000.

11. Zuckerman-Levin N, Tiosano D, Eisenhofer G, Bornstein S, Hochberg Z. The importance of adrenocortical glucocorticoids for adrenomedullary and physiological response to stress: a study in isolated glucocorticoid deficiency. J Clin Endocrinol Metab 2001;86(12):5920-5924. DOI: 10.1210/jcem.86.12.8106.

12. Arlt W, Society for Endocrinology Clinical Committee. Society for endocrinology endocrine emergency guidance: emergency management of acute adrenal insufficiency (adrenal crisis) in adult patients. Endocr Connect 2016;5(5):G1-G3. DOI: 10.1530/EC-16-0054. 